The greatest elastin antibody titer was noted in patients with SLE grade III (57.1\%, 4 people), with grade II - in 42.5\% (17 people), and with grade I - in 33.3\% (6 people).

The elastin antibody titer in SLE patients being admitted to hospital was reliably higher than in donors $(p<0.001)$.

An analysis of the findings showed that patients with vascular lesions demonstrated a significant elevation of elastin antibodies $(p<0.05)$.

Besides studying elastin antibodies, we analysed elastase antibodies. Elevated elastase antibodies were revealed in $72.2 \%$ of SLE patients with grade I of the condition; in $80.0 \%$ with grade II, and in $85.7 \%$ of patients with grade III. The titer of elastase antibodies in SLE patients admitted to hospital was reliably higher than in the control group $(p<0.01)$. The highest elastase antibody titer was noted when the skin and joints are affected, and vasculopathy is present.

Conclusions: The regularities revealed by pathogenetic method can be accounted for by considerable B-clonal expansion in SLE patients as the condition progresses. From the point of view of immunology, hyperproduciton of elastin and elastase autoantibodies is noted, which results in autoimmune lesion of the ligamentous apparatus, joints, skin and vessels where this protein is naturally present.

Disclosure of Interest: None declared

DOI: 10.1136/annrheumdis-2018-eular.2668

\section{AB0527 S100 PROTEINS ARE NOVEL BIOMARKERS FOR THE EFFICACY OF HCQ TREATMENT TO SKIN LESION IN SYSTEMIC LUPUS ERYTHEMATOSUS}

R. Wakiya, K. Ueeda, T. Kameda, S. Nakashima, M. Izumkawa, H. Shimada, A. Kondo, M. Kato, T. Miyagi, N. Kadowaki, H. Dobashi. Department of internal medicine, Division of hematology, rheumatology and respiratory medicine, Kagawa University, Kagawa, Japan

Background: Systemic lupus erythematosus(SLE) is deeply associated with not only acquired immunity but also innate immunity throughout toll like receptors (TLRs) signalling. Among many TLRs, TLR7 and TLR9 were reported to be closely associated with IFN- $\alpha$ production which contributed the pathogenesis of SLE. On the other hand, several reports demonstrated that S100A8 and S100A9 proteins which was known as one of damage-associated molecular patterns (DAMPs), were associated with disease activity of lupus nephritis. These proteins were also shown to reflect the treatment response by immunosuppressive therapy for SLE. ${ }^{12}$ However, there is no report about the effect of hydroxychloroquine (HCQ) on S100A8 and S100A9 proteins expression.

Objectives: To find a new biomarker of treatment with $\mathrm{HCQ}$, we focused on expression of S100A8 and S100A9 proteins in SLE.

Methods: We enrolled all SLE patients treated with HCQ in the absence of additional immunosuppressive therapy more than 3 months in our institute from Jan 2016 to Dec 2017 Serum levels of S100A8 and S100A9 proteins were measured by ELISA(CircuLex ELISA Kit, MBL) at the screening, 3 months and 6 months after HCQ administration. Disease activity of SLE was measured using the SLENA-SLEDAI 2011 Cutaneous disease activity was evaluated by Cutaneous Lupus Erythematosus Disease Area and Severity Index (CLASI). Immunological activity was examined by the levels of complement (C3, C4, CH50), anti-dsDNA anti-body and counting blood cell.

Results: 61 patients were enrolled in this study. HCQ was administered 48 cases with usual dose(based on ideal weight), 15 cases with low dose than usual dose (table 1).

Abstract AB0527 - Table 1

\begin{tabular}{lccc}
\hline HCQ dose & $\begin{array}{c}\text { Usual dose } \\
(\mathrm{n}=46)\end{array}$ & $\begin{array}{c}\text { Low dose } \\
(\mathrm{n}=15)\end{array}$ & $\mathrm{P}$ \\
\hline Age, years, mean $\pm \mathrm{SD}$ & $40 \pm 12$ & $46 \pm 9$ & 0.07 \\
Female, no.(\%) & $43(93)$ & $13(87)$ & 0.40 \\
Disease duration, & $13 \pm 10$ & $11 \pm 8$ & 0.61 \\
years & & & \\
skin lesion & $40(87)$ & $14(93)$ & 0.48 \\
renal lesion & $19(41)$ & $0(0)$ & $<0.001$ \\
SLEDAl score & $3.9 \pm 2.2$ & $2.7 \pm 1.8$ & 0.06 \\
anti-dsDNA, IU/ml & $15 \pm 17$ & $9.9 \pm 3.1$ & 0.91 \\
C3, mg/dl & $79 \pm 24$ & $87 \pm 26$ & 0.32 \\
C4, mg/dl & $16.1 \pm 7.6$ & $19.2 \pm 9.2$ & 0.30 \\
CH50, U/ml & $33.6 \pm 9.6$ & $37.3 \pm 7.4$ & 0.30 \\
CLASl activity & $3.6 \pm 3.2$ & $2.6 \pm 2.8$ & 0.11 \\
Prednisone & $41(89)$ & $15(100)$ & 0.11 \\
Dose, mg/day & $5.6 \pm 3.1$ & $8.1 \pm 5.1$ & \\
\hline
\end{tabular}

CLASI was improved by the treatment with HCQ independent of HCQ dose. However, the effect of HCQ on SLENA-SLEDAI and immunological biomarker was shown in the patients treated with usual dose $\mathrm{HCQ}$, not shown in those treated with low dose HCQ. On the other hand, serum levels of S100A8 and S100A9 proteins were significantly elevated in SLE patients with renal lesion $(p=0.02)$. These proteins were significantly decreased by the treatment with $\mathrm{HCQ}$ regardless of the $\mathrm{HCQ}$ dose $(\mathrm{p}<0.0001)$. The changes of serum S100A8 and S100A9 proteins during HCQ treatment(for 3 months) were significantly associated with changes of CLASI(figure 1).

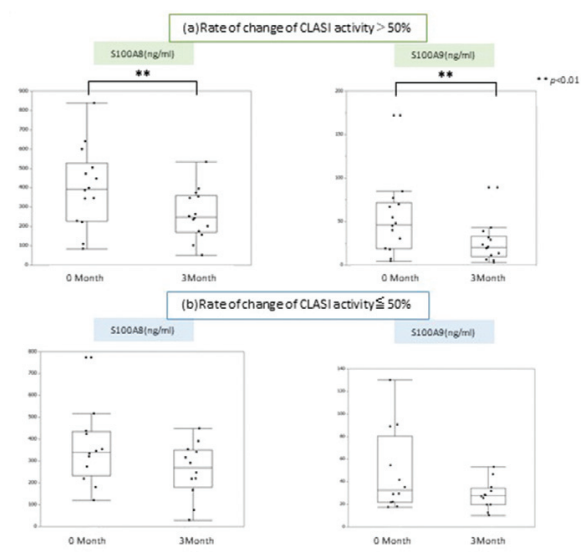

Abstract AB0527 - Figure 1. S100A8 and S100A9 proteins associated with CLASI score. Compared with SLE patients of $50 \%$ and less rate of change of CLASI activity, those of more than $50 \%$ rate of change of CLASI activity significantly decreased serum levels of S100A8 and S100A9 proteins after administration of HCQ. There were no SLE patients whose skin lesion got worse during HCQ treatment in this study.

Conclusions: HCQ reduced the expression of serum S100A8 and S100A9 pro teins, which reflected SLE disease activity especially in skin lesion. The measurement of S100A8 and S100A9 proteins is novel predictive biomarker for the efficacy of $\mathrm{HCQ}$ treatment on skin lesion in SLE patients.

\section{REFERENCES:}

[1] Tyden $\mathrm{H}$, et al. Pro-inflammatory S100 proteins are associated with glomerulonephritis and anti-dsDNA antibodies in systemic lupus erythematosus. Lupus 2017;(26):139-149.

[2] Tantivitayakul $P$, et al. Elevated expressions of myeloid-related proteins- 8 and -14 are danger biomarkers for lupus nephritis. Lupus 2016;(25):38-45

Disclosure of Interest: None declared

DOI: 10.1136/annrheumdis-2018-eular.2109

\section{AB0528 CONCOMITANT MEDICATION IN SYSTEMIC LUPUS ERYTHEMATOUS (SLE) PATIENTS TREATED WITH BELIMUMAB IN CLINICAL PRACTICE SETTINGS: RESULTS FROM THE OBSERVE STUDY IN SWITZERLAND}

J. von Kempis ${ }^{1}$, S. Dütsch ${ }^{2}$, N. Reuschling ${ }^{1,3}$, D. Schaer ${ }^{4}$, F. Vallelian ${ }^{4}$, R. Villiger ${ }^{5}$, P.M. Villiger ${ }^{5}$, R.B. Mueller ${ }^{1} .{ }^{1}$ Department of Rheumatology, Kantonsspital St. Gallen, St. Gallen; ${ }^{2}$ GlaxoSmithKline AG, Münchenbuchsee; ${ }^{3}$ Division of Rheumatology, Hirslanden, Basel; ${ }^{4}$ Universitä Hospital Zürich, Zürich; ${ }^{5}$ Department of Rheumatology, Inselspital Bern, Bern, Switzerland

Background: Systemic lupus erythematosus (SLE) is a complex and potentially severe autoimmune disease. Belimumab is the latest drug, and the first biologic compound, that was registered for treatment of SLE. There is limited information on safety and effectiveness of belimumab and its effects on the use of concomitant medication outside of randomised controlled trials.

Objectives: To describe the overall patterns of SLE care, outcomes and concomitant medication among belimumab users in Switzerland.

Methods: OBSErve Switzerland (GSK 201232) was a retrospective, multi-centre observational cohort study collecting data on the use of belimumab therapy in routine care of SLE Patients in Switzerland. SLE patients were included who had started therapy with $10 \mathrm{mg} / \mathrm{kg}$ belimumab/4 weeks at least six months before documentation. All patients were included regardless of treatment discontinuation during the study period. All statistical analyses were descriptive for both categorical and quantitative data.

Results: 53 SLE patients with belimumab as part of their routine treatment were analysed for this study. $81 \%$ of the patients were female, the mean age was 46.7 years and the mean BMI was $25.4 \mathrm{~kg} / \mathrm{m}^{2}$. The reasons to initiate belimumab were ineffective previous treatment $(66.0 \%)$, worsening of patient condition (28.3\%), and/or the intent to decrease glucocorticosteroid (GC) dose (47.2\%). 
During treatment, $58 \%$ of patients $(n=53$ ) showed an improvement of $\geq 20 \%$ and $23 \%$ of $\geq 50 \%$, based on physicians' evaluation of disease activity. This was consistent with an improvement of SELENA-SLEDAI (mean) from 8.0 at therapy start to 3.6 after six months on the basis of all available data $(n=27)$.

Similar outcomes were observed for arthritis, fatigue, rash, dsDNA antibody and complement levels.

Among the 42 patients treated with GC at time of belimumab initiation, GC dose was reduced by $5.7 \mathrm{mg} /$ day (mean) during treatment with belimumab (11.6 $\geq 5.9 \mathrm{mg} /$ day at six months). During the six months before initiation of belimumab, GC dose was stable or had to be increased in the majority of patients; however, during the six months therapy with belimumab, GC dose could be reduced in the majority of patients and GCs was discontinued in two patients. The percentages of patients receiving SLE co-medication other than GC were stable over the first six months of belimumab therapy. Within the first six months of treatment, no included patient had discontinued therapy with belimumab.

Conclusions: Treatment with belimumab over six months after initiation led to clinical improvement in a significant number of patients in real life settings and had an overall steroid-sparing effect. Belimumab was well-tolerated; no included patient discontinued the treatment within the first six months.

Acknowledgements: COMMERCIAL SUPPORT GRANT DISCLOSURE: Research funded by GlaxoSmithKline, UK.

Disclosure of Interest: J. von Kempis: None declared, S. Dütsch Employee of: SD is an employee of GSK, N. Reuschling: None declared, D. Schaer: None declared, F. Vallelian: None declared, R. Villiger: None declared, P. Villiger: None declared, R. Mueller: None declared

DOI: 10.1136/annrheumdis-2018-eular.5575

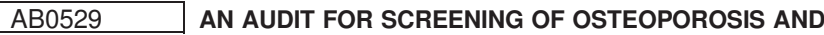 ITS MANAGEMENT IN PATIENTS WITH SYSTEMIC LUPUS ERYTHEMATOSUS}

S.A. Syed, K. Sunmboye, S. Shaffu. Rheumatology, University Hospitals of Leicester, NHS Trust, Leicester, UK

Background: Systemic lupus erythematosus (SLE) is an autoimmune, multi-system, chronic inflammatory condition. It is managed with long-term immunosuppressive therapy which includes steroid use. SLE is therefore considered an independent risk factor for osteoporosis.

Objectives: This clinical audit was undertaken to evaluate the screening of osteoporosis in patients diagnosed with SLE and assess adherence to national guidelines for the management of patients with low bone mineral density (BMD) on prolonged steroid therapy.

Methods: We studied 64 SLE patients seen at the Leicester Royal Infirmary. Demographic and clinical data was collected from the clinic letters. Steroid use for a cumulative period of over 4 weeks per year was considered significant and use of bisphosphonates or calcium and vitamin D supplements alone were taken into account for bone protection. BMD measurements by dual X-ray absorptiometry were performed. Osteoporosis was defined as a T score less than $-2.5 \mathrm{SD}$ in at least 1 region of measurement.

Results: Of the 64 patients studied, 54 (84.4\%) were female and 10 (15.6\%) male with an age range of 23 to 86 years and mean age of 47.45 years. Steroids were used in $46(71.9 \%)$ patients while $18(28.1 \%)$ patients did not receive any steroids. Twenty-one (32.8\%) patients had DEXA scans and whilst $43(67.2 \%)$ had not. Of those who had received steroids, 20 (43.4\%) patients underwent DEXA scans and 26 (56.5\%) did not. Osteoporosis was diagnosed in 3 (14.3\%) patients out of the 21 scanned. The therapies used are summarised in figure 1.

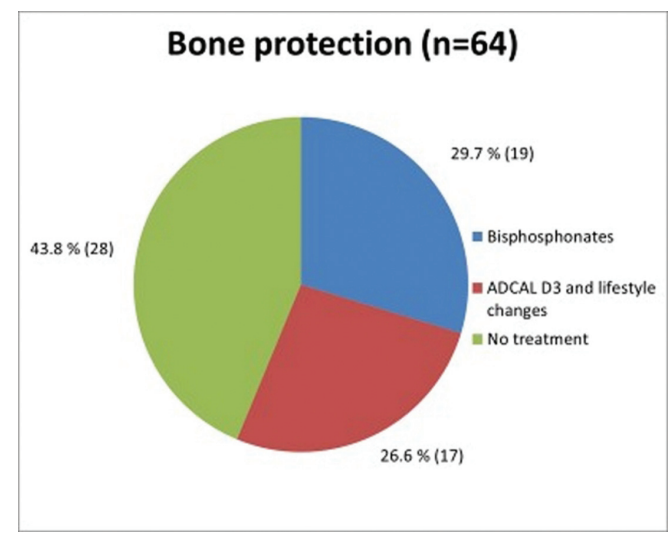

Abstract AB0529 - Figure 1. Bone protection $(n=64)$
Conclusions: Studies have shown that SLE is an independent risk factor for low BMD and use of corticosteroids is already a well-recognised risk for osteoporosis. Our study has shown that a large section of patients (43.8\%) did not receive any form of bone protection although, a significant proportion $(71.9 \%)$ were on oral steroids. Although a small section of those scanned demonstrated osteoporosis $(14.3 \%)$, many patients were already initiated on bisphosphonates without a DEXA. There was also no exclusion criteria set for young patients (age $<45$ years) or those who were newly diagnosed. Despite this, our study demonstrates the need for robust guidelines for the screening and management of bone health in patients with SLE in order to improve morbidity and mortality rates in this patient cohort.

\section{REFERENCES:}

[1] Mosca M,1 C Tani,1 M Aringer, et al. European League Against Rheumatism recommendations for monitoring patients with systemic lupus erythematosus in clinical practice and in observational studies.

[2] Almehed K, Forsblad d'Elia H, Kvist G, et al. Prevalence and risk factors of osteoporosis in female SLE patients-extended report. Rheumatology (Oxford) 2007;46:1185-1190.

[3] Bultink IE, Lems WF, Kostense PJ, et al. Prevalence of and risk factors for low bone mineral density and vertebral fractures in patients with systemic lupus erythematosus. Arthritis Rheum 2005;52:2044-2050.

[4] NOGG 2017: Clinical guideline for the prevention and treatment of osteoporosis.

Acknowledgements: We acknowledge the contribution of the clinical audit team at the University hospitals of Leicester NHS trust for their help in analysing the data.

Disclosure of Interest: None declared

DOI: 10.1136/annrheumdis-2018-eular.7336

\begin{tabular}{l|l}
\hline AB0530 & TREATMENT OUTCOME IN LUPUS NEPHRITIS \\
PATIENTS TREATED WITH MYCOPHENOLATE \\
MOFETIL: FROM REAL-WORLD CLINICAL PRACTICE
\end{tabular}

S.M. Jung ${ }^{1}$, D. Kim ${ }^{1}$, H.W. Kim² ${ }^{2}$, S.-W. Lee ${ }^{1}$, J.J. Song ${ }^{1}$, Y.-B. Park ${ }^{1}$. ${ }^{1}$ Internal Medicine, Yonsei University College of Medicine; ${ }^{2}$ Internal Medicine, Seoul National University Bundang Hospital, Seoul, Korea, Republic of Ireland

Background: Systemic lupus erythematosus (SLE) is a systemic autoimmune disease often characterised by the development of glomerulonephritis. ${ }^{1}$ There is a growing interest in the use of mycophenolate mofetil (MMF) as induction therapy and maintenance therapy for lupus nephritis. ${ }^{2,3}$

Objectives: This study aimed to evaluate the therapeutic outcome of MMF in lupus nephritis from real-world clinical practice, and identify the predictors for failure of remission after MMF treatment.

Methods: Korean patients with pathologically proven lupus nephritis class III, IV, and $\mathrm{V}$ were recruited from rheumatology clinic in Severance Hospital, Yonsei University College of Medicine between Nov 2011 and Aug 2017 Patients who treated with MMF for at least 3 months were included in the analysis. The probability of remission after MMF therapy, and the difference between patients who achieved remission or failed to achieve remission were analysed using KaplanMeier analysis and Cox proportional hazards model.

Results: Of 153 patients with lupus nephritis, 116 patients were included in this study. Seventy two patients continued MMF until the last follow-up. The mean age of patients was 34.2 years, and the median duration of SLE was 5.7 months. AntidsDNA antibody was positive in $82.8 \%$ of patients, and $9.5 \%$ of patients showed a histological class with pure $\mathrm{V}$ pathology. Mean protein/creatinine ratio in spot urine was 4.6 , and active urinary sediment was found in $82.8 \%$ of patients. During median follow-up period of 5 years, $80 \%$ of patients achieved clinical remission of lupus nephritis. Median time to remission was 4.2 months (IQR 0.9-9.1). Risk factors for failure of remission were nephrotic-range proteinuria and seronegativity of anti-dsDNA antibodies.

Conclusions: This study shows the real-world data on MMF treatment in patients with lupus nephritis. Patients with risk factors for failure to remission may require more intensive treatment and management.

REFERENCES:

[1] Yu F, Haas M, Glassock R, Zhao MH. Redefining lupus nephritis: clinical implications of pathophysiologic subtypes, Nature reviews. Nephrology 13 (2017) 483-495.

[2] Ginzler EM, Dooley MA, Aranow C, Kim MY, Buyon J, Merrill JT, et al. Mycophenolate mofetil or intravenous cyclophosphamide for lupus nephritis. The New England journal of medicine 2005;353(2005):2219-2228. 